\title{
Family Emotional Over-Involvement and Relapse among Inpatient Alcoholics in Nairobi, Kenya
}

\author{
Githae, Eunice Njango ${ }^{1}$, Sirera, Merecia ${ }^{2}$, \&Wasanga, Christine ${ }^{3}$ \\ 1,2,3 (Psychology Department: Kenyatta University, Kenya)
}

\begin{abstract}
Family interactional patterns have been proposed as significant in relapse of most psychiatric disorders. Many studies have demonstrated that patterns of interactions of family members affect the quality of relationships within such families. Research, particularly in Systems Theories, has also indicated that the ways in which family members interact with each other is cyclic and affect recovery and sustainability of treatment among psychiatric patients. Although the relationship between family communication dynamics and psychiatric relapse has received much attention, few studies have focused on such dynamics within alcoholic families. This study has examined one of the interactional patterns referred to as emotional over-involvement (EOI) among close family members with alcoholic patients. The study is underpinned by the Family Systems Theory (Minuchin, 1974), and views alcoholism as a 'family illness'. The study derived samples from inpatient alcoholics in rehabilitation centers $(\mathrm{N}=186)$ and their family members in Nairobi County, Kenya. The Alcohol Use Disorders Identification Test (AUDIT) was used to assess for alcoholism, while a questionnaire was generated to assess family EOI. Interviews were also carried out and were corroborated with the questionnaires. Pearson correlations and regression analyses showed that EOI was statistically significant at $\mathrm{p}=0.000<0.05$ and had a predictive value of .471. This meant that EOI expressed by family members was a very significant predictor for relapse among recovering alcoholics.
\end{abstract}

Keywords: emotional over-involvement, relapse, expressed emotion, addiction, rehabilitation/treatment, alcoholism, abstinence.

\section{INTRODUCTION}

The course of alcoholism has been related to many factors, some of which are found within the individual (Witkiewitz\&Marlatt, 2004) while others exist in the social milieu in which the alcoholic lives (Chan, 2003; Lipps, 1999). The individual and social support factors are seen as bidirectional, whereby each influences the other in a cyclic manner(Nichols \&Schwartz, 2006; Goldenberg \& Goldenberg, 2013). The family is seen to be the strongest social factor implicated in the course of alcoholism (De Maio, 1989). A supportive family environment is associated with better prognosis and successful reduction of drug use during treatment(Copello, Velleman, \& Templeton, 2005). There is strong evidence to support the effectiveness of family interventions in alcoholism treatment (Chan, 2003), which demonstrates that family therapy improves the overall quality of family relationships and functioning, which in turn improves the treatment outcome in substance use (Saatcioglu, Erim\&Cakmak, 2006; Beattie, 2001). However, family has also been implicated in inadvertently or involuntarily leading an individual family member to maladjusted behaviour such as drug use (Collins, 1990).

There appears to be dynamics within the family that are likely to contribute to instability within the recovering alcoholic that may result to relapse after treatment. Some family interactions that appear to be supportive may, in fact, promote relapse (Antoine, Christophe, \&Nandrino, 2009; Orford, Velleman, Copello, Templeton, \&Ibanga, 2010). Such interactions are mechanisms by family members to cope with the disorder, but become counterproductive and instead shield the addict from the negative consequences for his actions (Raitasalo\&Holmila, 2005). These coping mechanisms often termed as codependence have also been referred to as enmeshment according to Rotunda and O'Farrell (1998). This study therefore aimed at examining how some family interaction patterns end up contributing to a significant role in the course of alcoholism, particularly in relapse.

In his Family Systems Theory, Minuchin (1974) the concept of enmeshment seems to describe the patterns of behaviour depicted by emotional over-involvement. These are tightly interconnected relationships with unclear boundary lines. Minuchin described such families as too involved with each other to have clear lines of authority, and so entangled that leadership roles are diffuse in such families. Such enmeshment makes them over-react, give excessive concern to each other, and become intrusively involved with one another. EOI comprises of elements of overprotection, self-sacrifice and over caring attitudes (Hooley \&Gotlib, 2000). Families that have a member with a problem take it as their responsibility and obligation to take care and provide for this individual (Carlson, 2011; Collins, 1990). There are no clear boundaries of individual choices 
which may be compromised by the larger family needs. The family members over-identify and attend more to "their" shared identity and shared problems than those of each individual family member. Arguably, these kinds of relationships deny the individual opportunity to develop self capacities that can enhance their sobriety. The EOI concept describes relationships that are too overprotective and too self-sacrificing as those found in people who have enmeshed relationships (Rotunda \& O'Farrell, 1998) that leaves no space for individual growth.

The role of EOI in the course of psychiatric disorders has been extensively studied in areas such as suicide (Santos, Saraiva, \& De Sousa, 2009) Schizophrenia (e.g. Ng, Mui, Cheung, \& Leung, 2001; Ikram, Suhail, Jafery, \& Singh, 2011); obsessive-compulsive and agoraphobia (Chambless, \&Steketee, 1999), among others. Most studies seem to find a significant role of EOI in predicting and maintaining relapse in psychopathology (Hooley, 2007). Though EOI has not been studied as a single unidirectional concept, studies done in this area combine its role with other interactional dynamics within the family predicting relapse (e.g. Simmons, Chambless\& Gordon, 2008; Hooley \&Gotlib, 2000) and has been seen to predict and maintain relapse in psychopathology (L'Abate, 1998; Hooley, 2007).

Enmeshed families keep away from direct conflict by hindering each other from making independent decisions or by unending bickering, hence they are able to vent feelings without pressing for change or resolving issues ((Raitasalo\&Holmila, 2005; Hunter-Reel, McCrady, \& Hildebrandt, 2009). For the alcoholic family, this would mean that the shouting, anger and emotional climate would be a simple venting out of feelings, but such reactions are not necessarily to pressure the alcoholic to change their behaviour.

Another view of EOI is given by Kumar and Tiwari (2008), who argued that EOI from a mother had a likelihood of denying autonomy for children, which may nurture dependency. Literature on family and psychopathology shows that overprotection "momism" is predictive of psychopathology in children (Kumar \& Tiwari, 2008). When mothers offer too much care and protection, for instance by constantly keeping watch of them it may deprive the child many occasions to test reality and deny them opportunities to develop essential competencies (Kumar \& Tiwari, 2008).

Studies linked to EOI have demonstrated that an over-involved relative becomes so domineering that the patient feels someone else is in charge of their condition. They can no longer live with this kind of stress from pity and it makes them fall back into their illness as a way to cope (Hooley \&Gotlib, 2000). EOI has also been linked to relatives' interference in conversations during family discussions, behaviour that is verbally intrusive, and overstated statements and responses during patient- relative interactions (Fredman, Chambness\&Skeketee, 2004).

Though some studies have provided support for the association between family emotional overinvolvement (EOI) and relapse (e.g. Hooley, 2007;Pourmand, Kavanagh, \& Vaughan, 2005), literature shows that such information is however not consistent. Some findings are at odds with EOI-relapse association (e.g. Fichter, Glynn, Weyerer, Lieberman, \& Frick, 1997; O'Farrell, Hooley, Fals-Stewart, \& Cutter, 1998).). Most studies in this area have been done mainly with western populations, and mostly among populations with other psychopathologies rather than alcoholism. The central problem of this study was to therefore assess the relationship between the interaction patterns characterized by emotional involvement and its role in predicting relapse of the alcoholic.

\section{RESEARCH METHODOLOGY}

A correlation study was done to establish the relationship between family emotional over-involvement (EOI) and relapse among readmitted alcoholics in ten inpatient rehabilitation centers in Nairobi County, Kenya. Permission to conduct research was sought from program coordinators in the rehabilitation centers and the informed consent was sought from the participants. The participants were also assured of confidentiality. The study used both questionnaires and interviews to assess the existence of EOI within families of alcoholics. A total of 186 alcoholics and their close family members participated in the study. The inpatients were screened for alcohol addiction and were eligible for study if they were diagnosed to have alcoholism, which was assessed using the Alcohol Use Disorders Identification Test (AUDIT). Prior to readmission the alcoholic had to have lived with a close relative, or a family member equivalent, who was concerned about their addiction and who had more than 35 hours of face-face contact per week. Inpatient alcoholics responded to a questionnaire with 15 items assessing for EOI, which was also used to assess EOI of the family members with only a change in the pronoun -i.e. "I" for the alcoholic questionnaire and "he/she" for the family member questionnaire. The questionnaires demonstrated a reliability of 0.790 and, 0.789 respectively. $10 \%$ of each group was also interviewed and the interview data corroborated with the quantitative data derived from the questionnaires. Pearson correlations and regressions were used to test the study hypothesis: "There exists a relationship between EOI of family members and relapse of the recovering alcoholic". 


\section{RESULTS AND DISCUSSIONS}

Of the 200 questionnaires distributed to 10 rehabilitation centers, a total of 186 completed and usable questionnaires from the respondents representing a response rate of about $93 \%$ percent were returned. To address issues of possible common method variance, Cronbach Alpha reliability test was conducted for all the measures, which fell between 0.789 and 0.790 , and was hence satisfactory for study that is exploratory in nature. This indicated the reliability of the scales was reasonably high thus, depicting high internal consistency among the measurement items. Table 1 shows the demographic characteristics of the respondents.

Table 1: Demographic Characteristics of Respondents

\begin{tabular}{|c|c|c|}
\hline Characteristics & Frequency & Percentage \\
\hline \multicolumn{3}{|l|}{ Age of Respondents } \\
\hline Less than 18 & 4 & 2.2 \\
\hline $19-25$ years & 41 & 22.0 \\
\hline $26-40$ years & 107 & 57.5 \\
\hline $41-55$ years & 30 & 16.1 \\
\hline 56 and above & 2 & 2.2 \\
\hline Total & 186 & 100 \\
\hline \multicolumn{3}{|l|}{ Level of Education } \\
\hline Primary-Secondary School & 39 & 21 \\
\hline Diploma or Equivalent & 37 & 19.9 \\
\hline Bachelor's Degree or Equivalent & 106 & 57 \\
\hline Postgraduate & 4 & 2.1 \\
\hline Total & 186 & 100 \\
\hline \multicolumn{3}{|l|}{ Gender of Respondents } \\
\hline Female & 9 & 5 \\
\hline Male & 177 & 95 \\
\hline Total & 186 & 100 \\
\hline \multicolumn{3}{|l|}{ Marital Status } \\
\hline Single & 103 & 55.37 \\
\hline Married & 55 & 29.6 \\
\hline Separated/Divorced & 25 & 13.5 \\
\hline Widowed & 3 & 1.6 \\
\hline Total & 186 & 100 \\
\hline
\end{tabular}

Among the 186 respondents who participated in the study, the majority (57.5\%) were aged between 26 to 40 years, followed by those between 19 to 25 years (22\%), while those between $41-55$ years were $16.1 \%$. Therefore a total of $95.6 \%$ of respondents were aged between 19 to 55 years, which was an indication that majority of inpatients in rehabilitation centers in Nairobi Kenya were within the productive age. Majority of the respondents (79\%) possessed educational qualification higher than diploma or its equivalent. Of the respondents, $95 \%$ were male, while 5\% were female. About $55.4 \%$ were unmarried, while $44.6 \%$ had ever been married but were either divorced, separated or widowed. In general, the study indicated that majority of the respondents were unmarried $(70.4 \%)$, and the findings gave a favourable picture concerning the level of education of the respondents, which was an indication that the respondents are composed of highly educated people.

The working definition for the study relapse was "readmission to a rehabilitation center within the first year after treatment'. Participants were asked how many times they had been readmitted to a rehabilitation center within the past year. Among the participants, $13.4 \%$ had relapsed once within the first year, $17.7 \%$ had relapsed two times, and $4.8 \%$ had relapsed three times, while 3.2\% had relapsed more than three times during the first year after treatment, giving a total of $39.2 \%$ of cases of inpatient alcoholics that had relapsed and readmitted to the rehabilitation centers within the year after first treatment. This is shown in Table 2.

Table 2: No. of Readmissions for Inpatient Alcoholics

\begin{tabular}{|l|l|l|}
\hline No. of Readmissions & Frequency & Percent \\
\hline Once & 25 & 13.4 \\
\hline Twice & 33 & 17.7 \\
\hline Thrice & 9 & 4.8 \\
\hline More Than 3 Times & 6 & 3.2 \\
\hline Total & $\mathbf{7 3}$ & $\mathbf{3 9 . 2}$ \\
\hline
\end{tabular}




\begin{tabular}{|l|l|l|}
\hline Not readmitted & 113 & 60.8 \\
\hline Total & $\mathbf{1 8 6}$ & $\mathbf{1 0 0 . 0}$ \\
\hline
\end{tabular}

While statistics for readmission given by NSDUH (2006) in America indicated a relapse and readmission rate of over 50\% in the USA, this was much higher than the findings of this study. The difference may perhaps be explained by differences between the levels of awareness, availability of treatment, and perhaps government support provided for mental health in the different countries. Deveau, Tengia, Mutua,Njoroge, Dajoh, \& Singer, (2010) had indicated abstinence rate of $42 \%$ in Kenya for alcohol, and cannabis in four rehabilitation centers in Kenya. It is notable that the Kenya population is still characterized with stigma for mental health and a lack of awareness on where rehabilitation can be available in the country. Again, rehabilitation fee is high in most parts of the country and many people may relapse and are unable to continue with treatment within the rehabilitation centers in the country.

The researchers assessed the degree to which the respondents felt that the family members expressed emotional over-involvement (EOI) to the recovering alcoholic. EOI was measured by an instrument having 15 items each rated on a 5-point scale. The respondents were asked to indicate on a scale of 1 'strongly disagree' to 5 'strongly agree' whether the statements reflected how they felt about expression of EOI in the family. A sample question for the inpatient alcoholic was "My family doesn't like the way I take care of myself". Close family members also indicated how much they agreed with statements indicating how they were involved emotionally with their recovering family member. A sample question to measure the family member's emotional over-involvement was "I have sacrificed too much for him/her". The summary of responses is summarized in Figure 1.

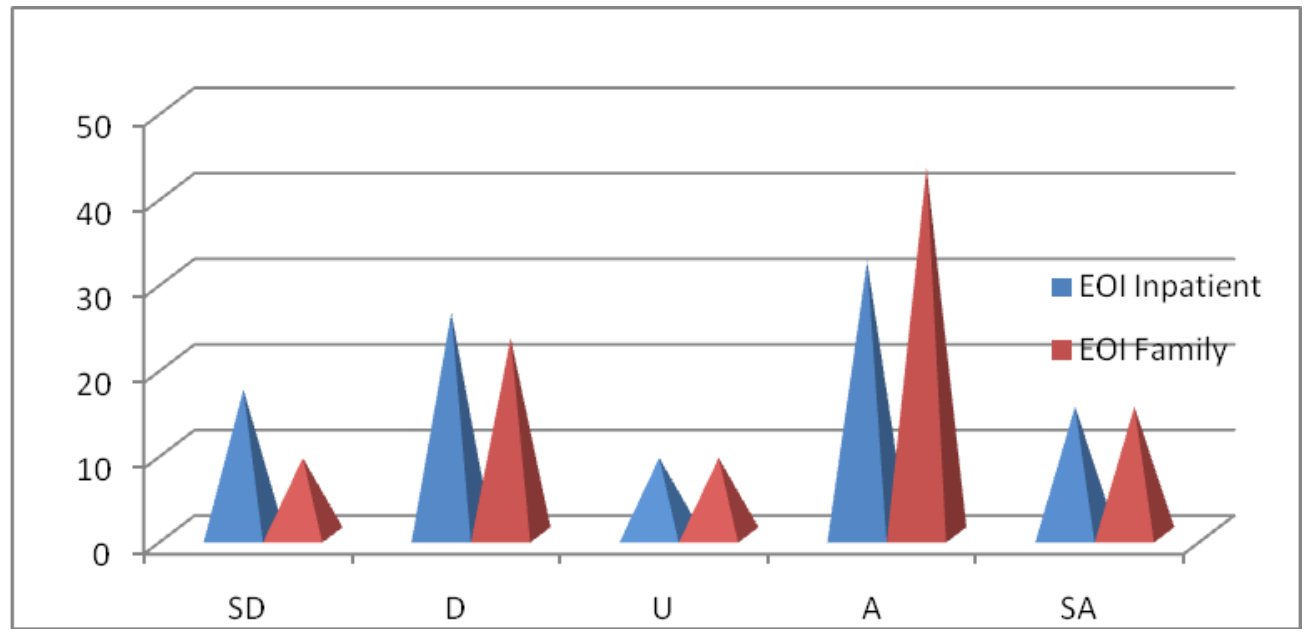

Figure 1.Emotional Over-involvement by Respondents.

According to the findings, both the inpatient alcoholics and their close family member indicated high levels of EOI. Both the inpatient alcoholics and their family members recorded high number of scores on the experience of EOI in their families as indicated by both 'agree' and 'strongly agree' responses. High levels of EOI were also supported by interviews with their close family members. The survey responses were corroborated with interview findings of family members. Some verbatim statements derived from interviews were as follows:

"This boy does not seem to learn..... What is annoying is that we make all the decisions for him because he just won't do it right..."

"I care so much for him that is why I do everything for him....."

"I don't think anyone else can care for him the same way that I have...."

"He can't survive without me around. He would kill himself with alcohol...."

"I have sacrificed everything for him. I am always available when he needs me. I have supported him in so many projects that he does not finish and I have paid for his rehabilitation three times. But he does not seem to see what I have been through...."

"I would want him to get a job but not too far away. This way I can monitor how he spends his time..."

In sum, there was agreement between the survey questions and interviews that majority of the family members indicated high levels of EOI. 
Test of Hypothesis: The null hypothesis for the study was "There was no relationship between family emotional over-involvement and relapse of the recovering alcoholic. As shown in Table 3, there was a strong and positive relationship between family EOI and relapse of the recovering alcoholic with a correlation .520 .

Table 3: Correlations between Relapse and EOI

\begin{tabular}{|c|c|c|c|}
\hline \multirow{4}{*}{ Relapse } & & Relapse & EOI \\
\hline & Pearson Correlation & 1 & $.520^{* * *}$ \\
\hline & Sig. (2-tailed) & & .000 \\
\hline & $\mathrm{N}$ & 179 & 177 \\
\hline \multirow[t]{3}{*}{ EOI } & Pearson Correlation & $.520^{* * *}$ & 1 \\
\hline & Sig. (2-tailed) & .000 & \\
\hline & $\mathrm{N}$ & 177 & 182 \\
\hline
\end{tabular}

Based on the confirmation of directionality shown after application of the Pearson correlation as shown on Table 3, there was sufficient evidence to fail to retain $\mathrm{H}_{0}$. The findings confirmed that relapse was positively associated with the EOI at $\mathrm{p}=0.000<.01$. This implied that when family EOI increased, the likelihood of relapse in a recovering alcoholic also increased. The nature and strength of EOI predictive value was further investigated using linear regression analysis as shown on Table 4.

Table 4: Model Coefficients ${ }^{\mathrm{a}}$

\begin{tabular}{|l|l|l|l|l|l|l|}
\hline \multirow{2}{*}{ Model } & \multicolumn{2}{|l|}{ Unstandardized Coefficients } & $\begin{array}{l}\text { Standardized } \\
\text { Coefficients }\end{array}$ & \multirow{2}{*}{ Sig. } \\
\cline { 3 - 5 } \multicolumn{2}{|l|}{} & B & Std. Error & Beta & & \\
\hline \multirow{2}{*}{1} & (Constant) & 50.416 & 3.421 & & 14.736 & .000 \\
\cline { 2 - 7 } & EOI & .850 & .106 & .520 & 8.047 & .000 \\
\hline \multicolumn{2}{|l|}{ a. Dependent Variable: Relapse } \\
\hline
\end{tabular}

As shown in Table 4, there is a strong and positive relationship between family EOI and relapse. The overall model was supported by data and the results suggest that EOI is a key factor in predicting relapse in alcoholism $\left(.520 \mathrm{p}=0.000<0.05\right.$. The adjusted $\mathrm{R}^{2}$ was .340 which implied that $34.0 \%$ of the variations in relapse are adequately explained by expressions of family EOI used in this study. While some studies in psychiatric illnesses have confirmed the EOI-relapse association, cultural understandings of EOI must be put into consideration when evaluating the EOI concept. $\mathrm{Ng}$, et al, (2001) opined that different degrees of EOI are considered normal or abnormal in different cultures. Cultural sensitivity and differences in the manifestation of psychological problems must be put into focus before extrapolating any studies as normal behavior patterns (Lopez, Ramirez, Ullman, Kopelowicz, Jenkins, Breitborde, \& Placenia, 2009).Culture in Kenya has evolved and traditional family structures have been disrupted by Westernization as people adopt more individualistic ways of living. However, it appears that family members who assume responsibility of those struggling with alcohol become either consciously or unconsciously over-involved in the healing process of the alcoholics. Reflecting on the qualitative data such as "I care so much for him that is why I do everything..." suggests that the EOI of family members dis-empowers the recovery alcoholic in developing capacities that can be of protective value in the healing process. This not only goes against what they are trained to do at the rehabilitationcenters but also pushes the recovering alcoholic to escapists behavior that can be counterproductive leading to relapse.

At the same time, a religious culture (which characterizes most Kenyans) promotes self-sacrifice in taking care of a loved one. This could mean that the emotionally over-involved family membertakes responsibility of alcoholism problem leaving the patients themselves with no responsibility to encourage change of behavior. This denies them motivation for change since there is someone to force change of behavior to happen to them. Therefore, although it is important to care for the recovering alcoholics, family members need to know that their overbearing could be counterproductive. Instead care that allows individual to test new behaviours aimed at maintaining sobriety should be encouraged. They should also understand that alcohol is addictive hence change is gradual. Patience and support in the little efforts made by the patient is important.

\section{CONCLUSION AND RECOMMENDATIONS}

The study made important findings relating to emotional over-involvement (EOI) and concluded that family EOI was a major predictor of relapse among recovering alcoholics. EOI is depicted by interaction dynamics within the family that seem to be comprised of elements of over-protection, self-sacrifice and too much caring for the alcoholic, which end up being destructive and may cause relapse. This study therefore 
recommends that treatment or rehabilitation efforts in addiction counseling focus on the interactional dynamics of family members. Treatment of alcoholism should consider reaching out to the families of alcoholics perhaps using community based interventions, where the doctor spends time with family members in their natural environments. The community rehabilitation would perhaps unearth faulty communication patterns and help family members re-learn and test new behaviours that would encourage the alcoholic maintain his/her sobriety goal. Perhaps families with an alcoholic would form social support groups and encourage them develop conversations to better improve their interaction skills. These support groups for families of alcoholics would replicate alcoholic anonymous (AA) support groups to help the "sick family" guard against relapsing into former interaction patters, particularly those characterized by EOI. More studies are required in investigating the role of EOI in relapse of individuals suffering alcohol dependency in wider contexts.

\section{REFERENCES}

[1] Witkiewitz, K., \&Marlatt, G. A. (2004). Relapse prevention for alcohol and drug problems. American Psychologist, 59(4), 224-235.

[2] Chan, J.G. (2003). An examination of family-involved approaches to alcoholism treatment.The Family Journal, 11(2), 129-138.

[3] Lipps, A.J. (1999). Family therapy in the treatment of alcohol related problems: A review of behavioural family therapy, family systems therapy, and treatment matching research. Alcoholism Treatment Quarterly, 17(3), 13-23.

[4] Nichols, M. P., \& Schwartz, R. C. (2006). Family therapy: Concepts and methods. New York: Pearson.

[5] Goldenberg, I., \& Goldenberg, H. (2013). Family therapy: An overview ( $8^{\text {th }}$ ed.).Belmont, CA: Waldsworth/Thomson Learning.

[6] De Maio, R. (1989). Integrating traditional alcoholic treatment programs and family-systems therapy, Family Systems Medicine, 7(3), 274-291.

[7] Copello, A.G., Velleman, R.D., \& Templeton, L. J. (2005). Family interventions in thetreatment of alcohol and drug problems. Drug Alcohol Review, 24(4), 369-385.

[8] Saatcioglu, O., Erim, R., \& Cakmak, D. (2006). Role of Family in alcohol and substance abuse. Review Article, Psychiatry and Neurosciences, 60(2), 125-132.

[9] Beattie, M. C. (2001). Meta-analysis of social relationships and post treatment drinking outcomes: Comparison of relationship structure, function and quality. Journal of Studies in Alcohol, 62(4), 518527.

[10] Collins, R.L. (1990). Family treatment of alcohol abuse: Behavioural systems perspective. In R.L. Collins, K.E. Leonard, \& J. S. Searles (Eds.).Alcohol and the family: Research and clinical perspectives (pp. 338-355). New York: Guilford.

[11] Antoine, P., Christophe, V., \&Nandrino, J. (2009). Crossed evaluations of temptation to drink, strain and adjustment in couples with alcohol problems. Journal of Health Psychology, 14(8), 1156-1162.

[12] [12] Orford, J., Velleman, R., Copello, A., Templeton, L., \& Ibanga, A. (2010). The experiences of affected family members: A summary of two decades of qualitative research. Drugs, Education, Prevention and Policy, 17(1), 44-62.

[13] [Raitasalo, K., \& Holmila, M. (2005). The role of the spouse in regulating one's drinking. Addiction Research and Theory, 13(2), 137-144.

[14] Rotunda, R.J., \& O'Farrell, T.J. (1998). Understanding and managing expressed emotion in the couple's treatment of alcoholism. Psychotherapy in Practice, 4(3), 55-71.

[15] Minuchin, S. (1974). Families and Family Therapy. Massachusetts: Harvard University Press, Cambridge.

[16] Hooley, J.M., \&Gotlib, I. H. (2000). A Diathesis-stress conceptualization of expressed emotion and clinical outcome.Applied and Preventive Psychology, 9(3), 135-151.

[17] Carlson, R. G. (2011). Communication deviance, expressed emotion, and family cohesion in schizophrenia. Open Access Dissertations, Paper 615.

[18] Santos, J. C., Saraiva, C. B., \& De Sousa, L. (2009). The role of expressed emotion, self- concept, Coping, and depression in parasuicidal behavior: A follow-up study. Archives of Suicide Research, 13, 358-367.

[19] Ng, R. M. K., Mui, J., Cheung, H. K., \& Leung, S. P. (2001). Expressed emotion and relapse of schizophrenia in Hong Kong. Hong Kong Journal of Psychiatry, 11(1), 4-11.

[20] Ikram, A., Suhail, K., Jeffery, S. Z., \& Singh, S. (2011). Rates of expressed emotion in Pakistan relatives of patients with schizophrenia. Israel Journal of Psychiatry and Related Sciences, 48(2), 74-81.

[21] Chambless, D.L., \&Steketee, G. (1999). Expressed emotion and behaviour therapy outcome. A prospective study with obsessive-compulsive and agoraphobic outpatients. Journal of Consulting and Clinical Psychology, 67(5), 658-665. 
[22] Hooley J. M. (2007). Expressed emotion and relapse of psychopathology.Annual Review of Clinical Psychology, 3, 329-352.

[23] Simmons, R. A., Chambless, D. L., \& Gordon, P. C. (2008). How do hostile and emotionally overinvolved relatives view relationships? What relatives' pronoun use tell us. Family Process, 47(3), 405-419.

[24] [L'Abate, L. (Ed.). (1998). Family psychopathology: The relational roots of dysfunctional behaviour. New York: Guilford Press.

[25] Hunter-Reel, D., McCrady, B., \& Hildebrandt, T. (2009). Emphasizing interpersonal factors: An extension of the Witkiewitz and Marlatt relapse model.Addiction, 104(8), 1281-1290.

[26] Kumar P. \& Tiwari, S. C. (2008). Family and psychopathology: An overview Series-1: Children and adults. Delhi Psychiatry Journal, 11 (2), 140-149.

[27] Fredman, S.J., Chambless, D.L., \&Steketee, G. (2004). Development and validation of an observational coding system for emotional-overinvolvement. Journal of Family Psychology, 18, 339-347.

[28] Pourmand, D., Kavanagh, D. J. and Vaughan, K. (2005), Expressed emotion as predictor of relapse in patients with comorbid psychoses and substance use disorder. Australian and New Zealand Journal of Psychiatry, 39(6), 473-478.

[29] Fichter, M. M., Glynn, S. M., Weyerer, S., Lieberman, R. P. \& Frick, U. (1997). Family climate and expressed emotion in the course of alcoholism. Family Process, 36(2), 203- 221.

[30] O'Farrell, T. J., Hooley, J., Fals-Stewart, W., \& Cutter, H. (1998). Expressed emotion and relapse in alcoholic patients. Journal of Consulting and Clinical Psychology, 66(5), 744-754.

[31] National Survey on Drug Use and Health (NSDUH, 2006). Substance Abuse and Mental Health Services Administration.

[32] Deveau, C. S., Tengia, L., Mutua, C., Njoroge, S., Dajoh, L., \& Singer, B. (2010).Utilization of community-based outpatient addiction treatment programmes in Kenya. African Journal of Drug and Alcohol Studies, 9(2), 62-70.

[33] Lopez, S. R., Ramirez, G. J., Ullman, J. B., Kopelowicz, A., Jenkins, J., Breitborde, N. J., \&Placenia, P. (2009). Cultural variability in the manifestation of expressed emotion. Family Process, 48(2), 179-194. 